\title{
Plastic deformation of titanium alloy Ti-6Al-4V in a complex stressed state under tension at high-strain rates
}

\author{
V. V. Skripnyak ${ }^{\dagger}$, K. V. Iohim, E. G. Skripnyak, V. A. Skripnyak \\ ${ }^{\dagger}$ skrp2012@yandex.ru \\ National Research Tomsk State University, Tomsk, 634050, Russia
}

\begin{abstract}
The article presents the results of a studies on mechanical behavior of titanium alloy Ti-6Al-4V (Ti Grade 5) in the range of strain rates from 0.1 to $10^{3} \mathrm{~s}^{-1}$. Tensile tests were carried out on flat and notched specimens using the Instron VHS 40/50-20 servo-hydraulic testing machine. High-speed video recording was carried out with a Phantom 711 Camera. The strain fields in the measuring area of the sample were investigated by the Digital Image Correlation (DIC) method. Analysis of the deformation fields of Ti-6Al-4V specimens under uniaxial tension at high strain rates revealed the presence of stationary localized shear bands at the initial stages of strain hardening. The evolution of the deformation fields in the studied loading regime indicates that in the localization bands the plastic strain is significantly higher than the average values in the gage zone of the specimen. It was found that the value of strain before fracture of the Ti-6Al-4V alloy in the zone of strain localization increases with the tension strain rate. The fracture of the titanium alloy is a result of cracks formation in the zone of plastic strain localization bands oriented along the surface of the action of the maximum shear stresses. The results obtained confirm the ductile nature of fracture of Ti-6Al-4V at strain rates from 0.1 to $10^{3} \mathrm{~s}^{-1}$, at the triaxiality stress parameter $0.33<\eta<0.44$, and at an initial temperature of $295 \mathrm{~K}$. At the same time, Ti-6Al-4V alloy demonstrates a tendency to embrittlement with an increase in the triaxiality stress parameter to 0.497 at the same loading condition.
\end{abstract}

Keywords: high strain rate deformation, fracture, strain localization, titanium alloys, stress triaxiality.

УДК: 539.5

\section{Пластическая деформация титанового сплава ВТ6 в сложном напряженном состоянии при высокоскоростном растяжении}

\author{
Скрипняк В. В. ${ }^{\dagger}$ Иохим К. В., Скрипняк Е. Г., Скрипняк В. А. \\ Национальный исследовательский Томский государственный университет, Томск, 634050, Россия
}

В статье представлены результаты исследования механического поведения титанового сплава BT6 (Ti-6Al-4V или Ti Grade 5) в диапазоне скоростей деформации от 0.1 до $10^{3} \mathrm{c}^{-1}$. Испытания на растяжение проводились на плоских сглаженных образцах и образцах с надрезом на сервогидравлической испытательной машине Instron VHS 40/50-20. Скоростная видеорегистрация проводилась камерой Phantom 711 Camera. Поля деформаций на измерительной площади образца исследовались методом корреляции цифровых изображений (DIC). Анализ полей деформаций в рабочей части образцов ВТ6 в условиях одноосного высокоскоростного растяжения выявил наличие стационарных полос локализованного сдвига на начальных стадиях деформационного упрочнения. Эволюция полей деформации в исследуемом режиме нагружения указывает на то, что в полосах локализации происходит существенно большая пластическая деформация по сравнению с усредненными значениями в рабочей части образцов. Установлено, что величина деформации до разрушения в зоне локализации деформации сплава ВТ6 увеличивается с ростом скорости деформации в исследуемых условиях нагружения. Разрушение сплава происходит за счет образования трещин в полосах локализации, ориентированных вдоль поверхности действия максимальных сдвиговых напряжений. Полученные результаты подтверждают вязкий характер разрушения ВТ6 при скоростях деформации от 0.1 до $10^{3} \mathrm{c}^{-1}$, при значениях параметра трехосности напряженного состояния $0.33<\eta<0.44$ и начальной температуре 295 К. При этом ВТ6 проявляет тенденцию к охрупчиванию с увеличением параметра трехосного напряжения до 0.497 при тех же условиях нагружения.

Ключевые слова: высокоскоростная деформация, разрушение, локализация деформации, титановые сплавы, трехосность напряженного состояния. 


\section{1. Введение}

Титановый сплав BT6 (Ti-6Al-4V) широко применяется для изготовления легких, надежных и коррозионностойких деталей механизмов и машин, элементов конструкций авиационно-космических и транспортных систем [1]. Несмотря на большой объем проведенных исследований, проектирование $3 \mathrm{D}$ изделий со сложной геометрической формой из сплава ВТ6, допускающих динамические воздействия при эксплуатации, представляет серьезную научно-техническую задачу. Сложности прогнозирования механического отклика титанового сплава ВТ6 обусловлены чувствительностью свойств К изменению фазового состава, зеренной структуры и текстуры при квазистатических и динамических нагрузках [1-3]. При деформировании сплава при комнатной температуре и скорости деформации $10^{-3} \mathrm{c}^{-1}$ с увеличением объемной доли первичной альфафазы предел текучести снижается, а деформация разрушения Ti-6Al-4V увеличивается [4]. Сплав в субмикрокристаллическом (СМК) и наноструктурном (НC) состоянии способен деформироваться в режиме сверхпластичности [5,6]. Однако при квазистатическом и динамическом сжатии деформация до разрушения сплава в СМК и НК состояниях снижается с уменьшением размеров зерна [7]. Анализ структуры деформированного сплава показал, что ограничение деформационного упрочнения и снижение деформации до разрушения обусловлено образованием систем полос локализованной деформации с последующим зарождением повреждений.

В [8] было обнаружено, что в микроструктуре крупнокристаллического сплава Ti-6Al-4V после квазистатической деформации при температуре ниже температуры рекристаллизации также имеются четко различимые области макро полос локализованной деформации, с заметными системами микрополос сдвига. В [9-12] было показано, что закономерности деформационного упрочнения, чувствительность напряжения течения к скорости деформации, предельная деформация до разрушения сплава в титановых сплавах ВТ1-0 и ВТ5-1 зависит от образования и эволюции полос локализации деформации.

В [13] было показано, что характеристики пластической деформации и разрушения $\mathrm{Ti}-6 \mathrm{Al}-4 \mathrm{~V}$ чувствительны как к скорости деформации, так и к трехосности напряжений, что обусловлено развитием локализованных сдвигов. В [14] было показано, что повышение ударной вязкости и удельной прочности слоистого композита на основе Ti-6Al-4V может быть достигнуто за счет целенаправленного формирования слоев с порами и повреждениями на границах раздела. В [15] приведены результаты, свидетельствующие о плавлении в зонах локализованного сдвига в сплаве Ti-6Al-4V в условиях динамического сжатия при начальной температуре 295 К. Локализация пластической деформации в титановых сплавах существенно влияет на механизмы зарождения и развития повреждений, вследствие чего, может рассматриваться в качестве фактора, который усложняет прогноз развития деформаций вблизи концентраторов напряжения.
Поэтому, получение более полной информации о закономерностях локализации пластической деформации при квазистатическом и высокоскоростном растяжении титанового сплава ВТ6 (Ti-6Al-4V) остается актуальной проблемой. Ее решение будет способствовать повышению точности прогнозов ресурса ответственных конструкций из сплава ВТ6 при динамических нагрузках, а также получению требуемых прочностных и деформационных свойств сплава в результате целенаправленного изменения его структуры.

Цель данной работы состояла в получении новых экспериментальных данных о закономерностях развития пластического течения и деформации сплава ВТ6 при растяжении в диапазоне скоростей деформации от 0.1 до $10^{3} \mathrm{c}^{-1}$.

\section{2. Материал и условия эксперимента}

Закономерности механического поведения сплава ВТ6 были исследованы при растяжении в диапазоне скоростей деформации от 0.1 до $10^{3} \mathrm{c}^{-1}$ при комнатной температуре. Съемка микроструктуры и определение химического состава образцов проводились с помощью сканирующего электронного микроскопа Tescan Vega TS 5130 MM, оснащенного энергодисперсионным спектрометром LINK (Oxford Instruments).

Сплав имел химический состав в вес.\%: Тi $\approx 90.64$; $\mathrm{Al} \approx 5.95 ; \mathrm{V} \approx 3.31$ и находился в поликристаллическом состоянии со средним размером зерна $\approx 25$ мкм.

Структура сплава ВТ6 (см. Рис. 1а) состоит из комбинации равноосных зерен альфа фазы с гексагональной плотноупакованной (ГПУ) решеткой и зерен с пластинчатой структурой, образованной пластинами альфа и бета фаз. Бета фаза имеет объемно центрированную кубическую (ОЦК) решетку.

Образцы были вырезаны из тонколистового проката сплава ВТ6 электроэрозионным методом. Толщина образцов $d$ составляла $1.1 \pm 0.01$ мм, а наименьшая ширина $w-6$ мм. Начальная длина рабочей части $l$ была равна $20 \pm 0.1$ мм. На Рис. 1b показана геометрия образцов, применявшихся при испытаниях. Минимальная площадь поперечного сечения плоских образцов $(w \times d)$ cocтавляла $A_{0}=6.6 \pm 0.06 \mathrm{mм}^{2}$. Испытания проведены в соответствии со стандартом ISO 26203-2:2011 на сервогидравлическом испытательном стенде Instron VHS 40/50-20.

Растягивающее усилие и смещения регистрировали с высоким временным разрешением вплоть до разрушения образца.

Для каждого типа образца были проведены по три испытания при каждой из скоростей деформации $10^{3}, 10^{2}$ и $0.1 \mathrm{c}^{-1}$. В каждой серии испытаний наблюдалась высокая степень воспроизводимости скорости деформации, усилий и смещений. Поля деформации образцов при растяжении были получены методом корреляции цифровых изображений (Digital Image Correlation - DIC) [16]. Для регистрации изменения геометрии образца использовалась камера Phantom V711 (Vision ResearchAMETEK Cо., Уэйн, Нью-Джерси, США) со скоростью $10^{5}$ кадров в секунду. Видео было записано в нескольких разрешениях: $1280 \times 800,1024 \times 680$ и $512 \times 400$ пикселей 


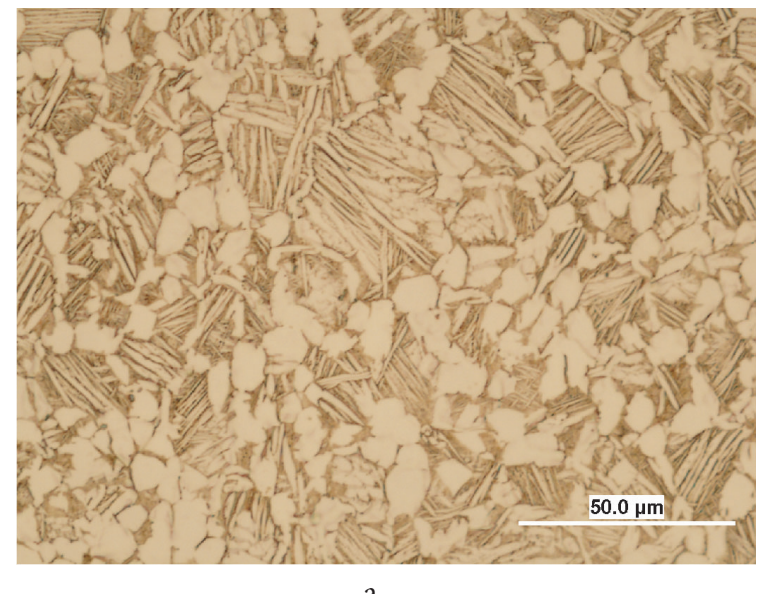

a
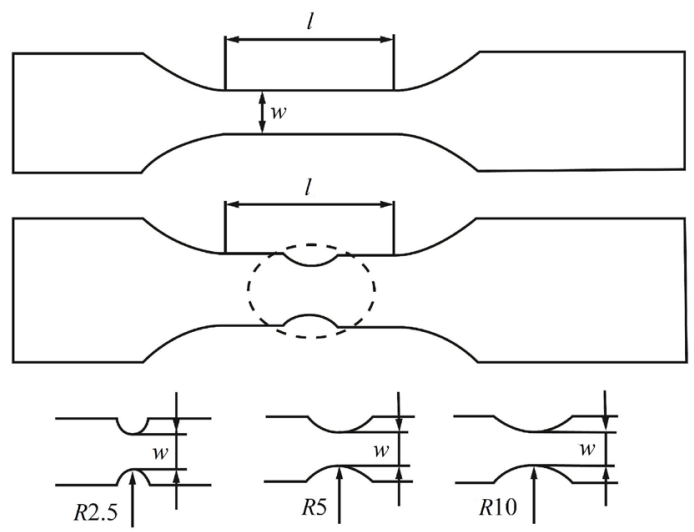

b

Pис. 1. (Color online) Микроструктура сплава ВТ6 (a); геометрия образцов (b).

Fig. 1. (Color online) Microstructure of Ti-6Al-4V alloy (a); samples geometry (b).

при скоростях деформации $10^{3}, 10^{2}, 0.1 \mathrm{c}^{-1}$. Размер изображения варьировался в зависимости от допустимого разрешения при высокоскоростной съемке деформируемых образцов. Были использованы размеры изображения с 250 пикселями вдоль минимальной ширины измерительной части образца.

Анализ полей деформаций методом DIC в рабочей зоне образца позволяет выбрать размер и положение виртуального экстензометра на рабочей части образца в зоне локализации пластического течения [16]. Это позволяет повысить точность и адекватность получаемых диаграмм истинное напряжение - истинная деформация. Размер виртуального экстензометра выбирался так, чтобы деформация была равномерной по его длине до момента времени, соответствующего появлению финальной нисходящей ветви (предразрушения) на диаграмме усилие-перемещение.

Истинное напряжение $\sigma_{1}^{\text {true }}$ и истинная деформация $\varepsilon_{1}^{\text {true }}$ для образцов с гладкой рабочей частью определялись по формулам [17]:

$$
\begin{gathered}
\sigma_{1}^{\text {true }}=\left(F / A_{0}\right)\left(1-\Delta L / L_{0}\right) \\
\varepsilon_{1}^{\text {true }}=\ln \left(1-\Delta L / L_{0}\right)
\end{gathered}
$$

где $F-$ растягивающее усилие, $A_{0}-$ начальная минимальная площадь поперечного сечения образца,
$\Delta L$ - удлинение виртуального экстензометра и $L_{0}-$ начальная длина виртуального экстензометра.

Параметр трехосности напряженного состояния $\eta$ определялся по формуле [9]:

$$
\eta=-p / \sigma_{\text {eq }}
$$

где $p=-\left(\sigma_{11}+\sigma_{22}+\sigma_{33}\right) / 3-$ гидростатическое давление, $\sigma_{\text {eq }}=\left[(3 / 2)\left(\sigma_{i j}-p \delta_{i j}\right)\left(\sigma_{i j}-p \delta_{i j}\right)\right]^{1 / 2}-$ эквивалентное напряжение, $\sigma_{i j}$ - компоненты тензора напряжения, $\delta_{i j}-$ символ Кронекера, $i, j=1,2,3$.

Начальная величина параметра трехосности напряженного состояния $\eta$ для плоских образцов с надрезами рассчитывалась по аналитической формуле для плоского напряженного состояния [18]:

$$
\eta=(1+2 A) /\left(3 \sqrt{A^{2}+A+1}, A=\ln [1+w /(4 R)],\right.
$$

где $w$ - минимальная ширина образца в зоне надреза, $R-$ радиус надреза.

Для гладких образцов $\eta=0.333$, для образцов с радиусами надрезов 2.5, 5 и 10 мм начальные значения $\eta$ имели значения $0.4973,0.4405,0.39612$, соответственно.

Эквивалентная пластическая деформация $\varepsilon_{\mathrm{eq}}^{\mathrm{p}}$ в случае одноосного наряженного состояния при растяжении плоских образцов определялась с учетом $\varepsilon_{2}^{\mathrm{p}}=\varepsilon_{3}^{\mathrm{p}}=-(1 / 2) \varepsilon_{1}^{\mathrm{p}}$ по формуле:

$$
\begin{gathered}
\varepsilon_{1}^{\mathrm{p}}=\varepsilon_{1}^{\text {true }}-\sigma_{1}^{\text {true }} / E, \\
\varepsilon_{\text {eq }}^{\mathrm{p}}=(\sqrt{2} / 3)\left[\left(\varepsilon_{1}^{\mathrm{p}}-\varepsilon_{2}^{\mathrm{p}}\right)^{2}+\left(\varepsilon_{2}^{\mathrm{p}}-\varepsilon_{3}^{\mathrm{p}}\right)^{2}+\left(\varepsilon_{3}^{\mathrm{p}}-\varepsilon_{1}^{\mathrm{p}}\right)^{2}\right]^{1 / 2},
\end{gathered}
$$

где $E$ - модуль Юнга, $\varepsilon_{i}^{\mathrm{p}}$ - главные компоненты тензора пластических деформаций.

Эквивалентная деформация в случае одноосного напряженного состояния определялась с учетом:

$$
\varepsilon_{\mathrm{eq}}=\left(\sigma_{\mathrm{eq}} / E\right)+\varepsilon_{\mathrm{eq}}^{\mathrm{p}} .
$$

Усредненная скорость деформации $\dot{\varepsilon}$ определялась по формуле [19]:

$$
\dot{\varepsilon}_{1}=v_{1}(t) / l
$$

где $v_{1}(t)-$ скорость перемещения точек рабочей части образца вдоль оси растяжения, $t-$ время.

\section{3. Результаты и обсуждение}

Зависимости истинных напряжений от истинных деформаций сплава ВТ6 для скоростей деформации $10^{3}, 10^{2}$ и $0.1 \mathrm{c}^{-1}$, полученные при одноосном растяжении плоских образцов ВТ6 с постоянным сечением рабочей части $(\eta=0.33)$, показаны на Рис. 2 а Напряжение пластического течения и предельные удлинения до разрушения сплава ВТ6 демонстрируют чувствительность к скорости деформации. Результаты показали, что с увеличением скорости деформации степень макроскопической однородной деформации в образцах уменьшается. На диаграммах истинное напряжение-истинная деформация метки указывают момент начала локализации пластической деформации (см. Рис. 2 a). При скоростях деформации выше $10^{2} \mathrm{c}^{-1}$ 
в образцах без надрезов наблюдалось формирование системы квазистационарных полос локализации уже при относительных удлинениях $\approx 0.07$. Отметим, что удлинения, соответствующие началу нисходящих ветвей диаграмм деформирования, существенно превышают удлинения, при которых формируются квазистационарные полосы локализации деформации (см. Рис. 2 a). С ростом времени растяжения в полосах локализации происходит увеличение $\varepsilon_{1}^{\text {true }}$, при этом положение полос на рабочей части образца остается постоянным. Вследствие увеличения локализованной пластической деформации в полосах происходит локальный разогрев до температуры, существенно превышающей среднюю температуру в рабочей части образца. Локальный разогрев в полосах локализованного сдвига препятствует зарождению и росту пор на микроуровне, вследствие чего степень деформации до разрушения увеличивается, а чувствительность к значению макроскопического параметра трехосности напряженного состояния $\eta$ уменьшается. На Рис. 2 b показаны диаграммы перемещение - усилие для гладких образцов и образцов с надрезами, имевших одинаковые минимальные начальные площади сечения. Повышение усилия деформирования при эффективной скорости
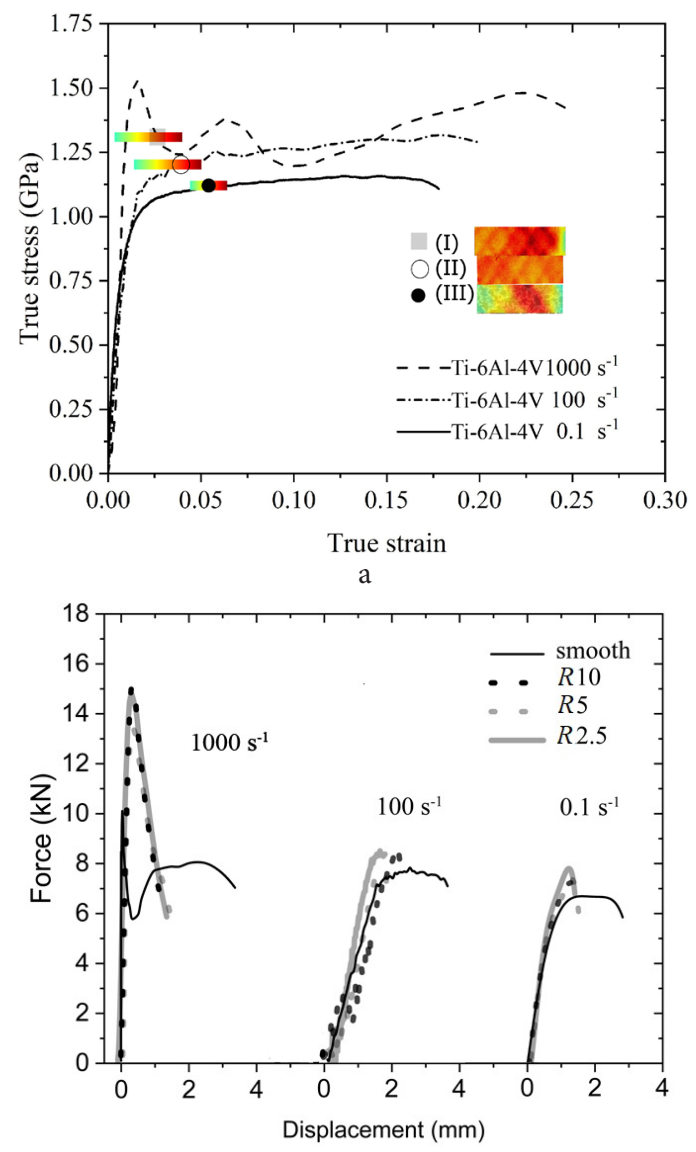

b

Puc. 2. (Color online) Зависимости истинных напряжений от истинных деформаций при растяжении плоских (a), зависимости усилия от перемещений (b) образцов ВТ6 со скоростями деформации $10^{3}, 10^{2}$ и $0.1 \mathrm{c}^{-1}$.

Fig. 2. (Color online) True stresses versus true strains curves under tension of flat specimens (a) and Force-Displacement diagrams (b) of Ti-6Al-4V at strain rates of $10^{3}, 10^{2}$ и $0.1 \mathrm{~s}^{-1}$. деформации $1000 \mathrm{c}^{-1}$ связано с торможением фронтов Людерса вследствие возникновения градиента эквивалентного напряжения в области переменного сечения образцов.

На Рис. 3 показаны поля эквивалентной деформации в рабочей части образцов, полученные методом DIC, в момент времени, предшествующий началу разрушения. Результаты свидетельствуют о том, что предельные степени деформации до разрушения сплава ВТ6 зависят от вида напряженного состояния и скорости деформации. Для гладких образцов и образцов с радиусами надрезов 5 и 10 мм максимальная степень деформации достигается в зоне пересечения сопряженных полос локализации пластической деформации в центре рабочей части, что указывает на вязкое разрушение. Отметим, что при значениях параметра трехосности напряженного состояния $\approx 0.4973$ изменяется характер распределения деформации в рабочей части образцов: максимальные деформации сосредоточены в зоне концентратора напряжений. Это свидетельствует о переходе от вязкого разрушения сплава ВТ6 к квазихрупкому. Отметим, подобной смены характера разрушения в диапазоне скоростей деформации от 0.1 до $10^{3} \mathrm{c}^{-1}$ в альфа титаном сплаве ВТ5-1 (Ti-5Al-2.5Sn) обнаружено не было [9].

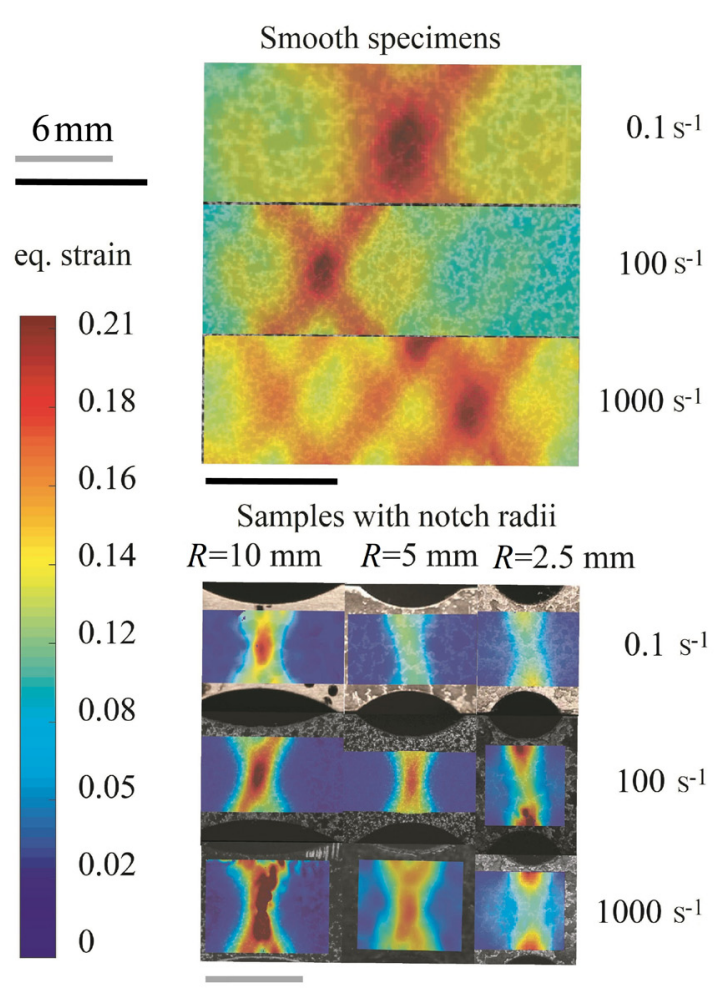

Pис. 3. (Color online) Эквивалентные деформации в гладких образцах при растяжении со скоростями деформации $10^{3}, 10^{2}$ и $0.1 \mathrm{c}^{-1}$, эквивалентные деформации в образцах с радиусами надрезов 10, 5 и 2.5 мм при растяжении со скоростями деформации $10^{3}, 10^{2}$ и $0.1 \mathrm{c}^{-1}$.

Fig. 3. (Color online) Equivalent strains in smooth specimens under tension with strain rates of $10^{3}, 10^{2}$ и $0.1 \mathrm{~s}^{-1}$, equivalent strain in specimens with notch radii of 10,5 and $2.5 \mathrm{~mm}$ under tension at strain rates of $10^{3}, 10^{2}$ и $0.1 \mathrm{~s}^{-1}$. 
Полученные результаты указывают, что в зоне формирования полос макроскопической локализации пластической деформации зарождаются повреждения на более низком масштабном уровне, сопоставимом с размерами зерен. Коалесценция повреждений при дальнейшем нагружении приводит к образованию трещин. На Рис. 4 показаны фотографии зон формирования трещин в плоских образцах ВТ6 и образцах с надрезом $(\eta=0.4973)$ после растяжения со скоростью деформации $10^{2} \mathrm{c}^{-1}$.

Ориентация сопряженных полос локализации в зоне шейки гладких образцов и в образцах с надрезами отличается. С ориентацией стационарных макроскопических полос локализации совпадает ориентация формирующихся трещин. Максимальные эквивалентные деформации в полосах перед зарождением трещин уменьшаются с ростом параметра трехосности напряженного состояния (см. Рис. 3). При этом из-за интенсификации локализации пластического течения с ростом скорости деформации наблюдается рост эквивалентной деформации в центральной области образцов. В зависимости от скорости деформации наблюдается существенное расхождение величин истинных деформаций при близких значениях усредненных удлинениях образцов.

Таким образом, скорость предварительной деформации и тип напряженного состояния можно отнести к важным факторам предыстории нагружения, определяющим развитие разрушения при растягивающих нагрузках сплава ВТ6.
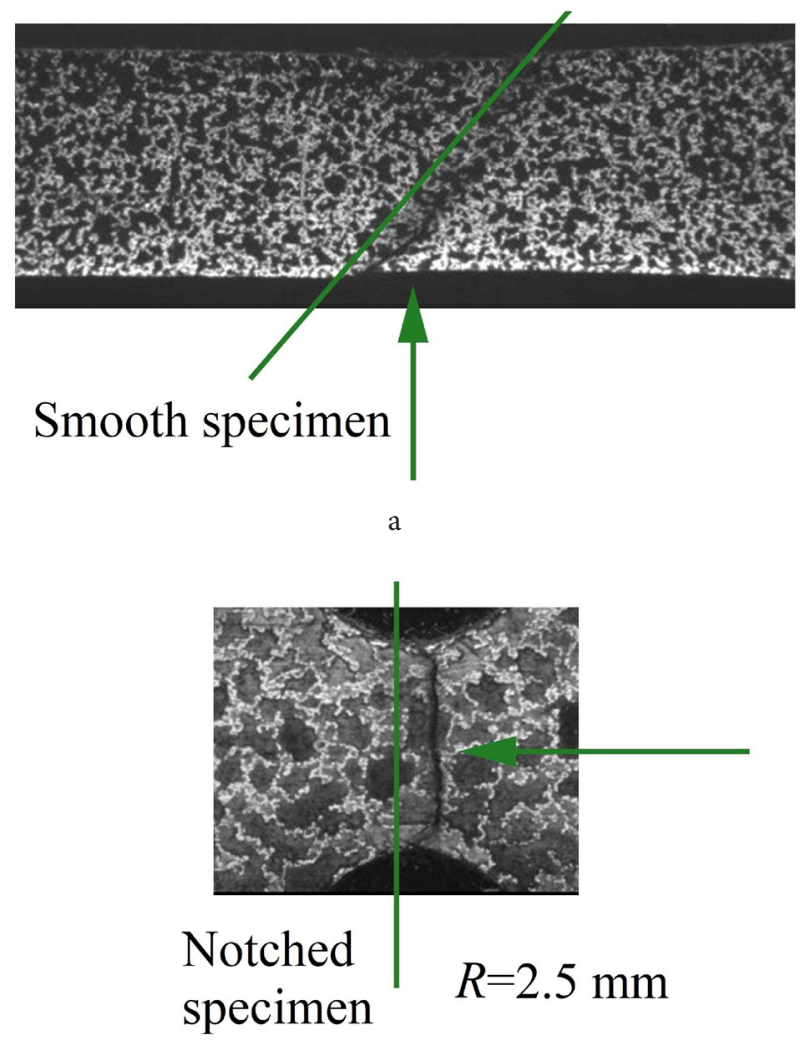

b

Рис. 4. Трещина в гладком образце после растяжения со скоростью деформации $10^{2} \mathrm{c}^{-1}$ (a), трещина в образце с надрезом (b).

Fig. 4. Crack in a smooth specimen after tension at a strain rate of $10^{2} \mathrm{~s}^{-1}(\mathrm{a})$, crack in a notched specimen (b).

\section{4. Заключение и выводы}

Представлены результаты экспериментов по растяжению титанового сплава ВТ6 в диапазоне скоростей деформации от 0.1 до $10^{3} \mathrm{c}^{-1}$ в условиях простого и сложного напряженного состояния.

Определены зависимости истинных напряжений и истинных деформаций при испытаниях сплава ВТ6 со скоростями деформации $10^{3}, 10^{2}$ и $0.1 \mathrm{c}^{-1}$ и при комнатной температуре.

Установлено, что сплав ВТ6 при увеличении параметра трехосности напряженного состояния в диапазоне от 0.44 до 0.497 происходит переход от вязкого разрушения к квазихрупкому.

Результаты исследования указывают на то, что скорость предварительной деформации и параметр трехосности напряженного состояния являются определяющими факторами, влияющими на предельные характеристики сплава ВТ6 и требующими учета для получения адекватного прогноза ресурса изделий из этого сплава.

Анализ полей деформаций в рабочей части образцов BT6 в условиях одноосного высокоскоростного растяжения выявил наличие стационарных полос локализованного сдвига на начальных стадиях деформационного упрочнения.

Установлено, что величина деформации до разрушения в зоне локализации деформации сплава ВТ6 увеличивается с ростом скорости деформации в исследуемых условиях нагружения.

Результаты исследования указывают на то, что скорость предварительной деформации и тип напряженного состояния относятся к факторам предыстории нагружения, определяющим развитие разрушения при растягивающих нагрузках и должны быть учтены для получения адекватного прогноза ресурса изделий из сплава ВТ6.

Благодарности / Acknowledgements. Работа выполнена при поддержке гранта Российского научного фонда № 20-79-00102. / This work was supported by the Russian Science Foundation, grant No. 20-79-00102.

\section{Литература/References}

1. M. Peters, C. Leyens. Titanium and Titanium Alloys. Weinheim, Wiley-VCH (2003) 513 p.

2. T. B. Britton, F. P. E. Dunne, A. J. Wilkinson. Proceedings of the Royal Society A: Mathematical, Physical and Engineering Science. 471 (2178), 20140881 (2015). $\underline{\text { Crossref }}$ 3. Z. $\mathrm{Xu}, \mathrm{X} . \mathrm{He}, \mathrm{H}$. Hu, P. Tan, Y. Liu, F. Huang. Int. J. Impact Eng. 130, 281 (2019). $\underline{\text { Crossref }}$

4. Y. Ren, S. Zhou, W. Luo, Z. Xue, Y. Zhang. IOP Conference Series: Materials Science and Engineering. 322, 022022 (2018). . Crossref

5. R. Ya. Lutfullin, A.A. Kruglov, R.V. Safiullin, M.K. Mukhametrahimov, O.A. Rudenko. Materials Science and Engineering A. 503, 52 (2009). Crossref

6. R. Y. Lutfullin, M. K. Mukhametrakhimov. Met. Sci. Heat. Treat. 48, 54 (2006). $\underline{\text { Crossref }}$ 
7. Y. Long, W. Zhang, L. Peng, H. Peng, X. Li, X. Huang. Metall. Mater. Trans. A. 51, 4765 (2020). Crossref

8. S. Roy, S. Suwas. J. Alloys Compd. 548, 110 (2013). Crossref

9. V. V. Skripnyak, E. G. Skripnyak, V.A. Skripnyak. Metals. 10 (3), 305 (2020). Crossref

10. V.A. Skripnyak, V.V. Skripnyak, E.G. Skripnyak, N. V. Skripnyak. Int. J. Mech. Mater. Design. 16, 215 (2020). Crossref

11. Yu.P. Sharkeev, V.P. Vavilov, O.A. Belyavskaya, V.A. Skripnyak, D.A. Nesteruk, A.A. Kozulin, V.M. Kim. J. Nondestr. Eval. 35, 42 (2016). Crossref

12. Yu.P. Sharkeev, V.P. Vavilov, V.A. Skripnyak, V.A. Klimenov, O.A. Belyavskaya, D.A. Nesteruk, A. A. Kozulin, A. I. Tolmachev. Russ. J. Nondest. 47 (10), 701 (2011). $\underline{\text { Crossref }}$
13. J. Huang, Y. Guo, D. Qin, Z. Zhou, D. Li, Y.Li. Theor. Appl. Fract. Mech. 97, 48 (2018). Crossref

14. C.M. Cepeda-Jiménez, F. Carreño, O.A. Ruano, A. A. Sarkeeva, A. A. Kruglov, R. Lutfullin. Mater. Sci. Eng. A. 563, 28 (2013). Crossref

15. S. Nemat-Nasser, W.G. Guo, V.F. Nesterenko, S.S. Indrakanti, Y.B. Gu. Mech Mater. 33, 425 (2001). Crossref

16. J. Blaber, B. Adair, A. Antoniou. Ncorr. Exp. Mech. 55, 1105 (2015). Crossref

17. Y. Bai, T. Wierzbicki. Int. J. Plast. 24, 1071 (2008). Crossref

18. Y. Bai, T. Wierzbicki. J. Eng. Mater. Technol. 131, 021002 (2009). Crossref

19. H. Hu, Z. Xu, W. Dou, F. Huang. Int. J. Impact Eng. 145, 103689 (2020). $\underline{\text { Crossref }}$ 